\title{
A METHODOLOGICAL APPROACH TO BISDN SIGNALLING PERFORMANCE
}

\author{
X. HOU \\ Centre for Telematics and Information Technology, University of Twente, \\ P.O. Box 217, 7500 AE Enschede, The Netherlands \\ N. D. KALOGEROPOULOS AND M. E. LEKKOU \\ Electrical and Compuser Engineering Department, National Technical University of Athens, Greece \\ I.G. NIEMEGEERS \\ Centre for Telematics and Information Technology, University of Twente, \\ P.O. Box 217, 7500 AE Enschede, The Netherlands \\ AND \\ I. S. VENIERIS \\ Electrical and Computer Engineering Department, National Technical University of Athens, Greece
}

\begin{abstract}
SUMMARY
Sophisticated signalling protocols are required to properly handle the complex multimedia, multiparty services supported by the forthcoming BISDN. The implementation feasibility of these protocols should be evaluated during their design phase, so that possible performance bottlenecks are identified and removed. In this paper we present a methodology for evaluating the performance of BISDN signalling systems under design. New performance parameters are introduced and their network-dependent values are extracted through a message flow model which has the capability to describe the impact of call and bearer control separation on the signalling performance. Signalling protocols are modelled through a modular decomposition of the seven OSI layers including the service user to three submodels. The workload model is user descriptive in the sense that it does not approximate the direct input traffic required for evaluating the performance of a layer protocol; instead, through a multi-level approach, it describes the actual implications of user signalling activity for the general signalling traffic. The signalling protocol model is derived from the global functional model of the signalling protocols and information flows using a network of queues incorporating synchronization and dependency functions. The same queueing approach is followed for the signalling transfer network which is used to define processing speed and signalling bandwidth requirements and to identify possible performance bottlenecks stemming from the realization of the related protocols.
\end{abstract}

KEY woRDS Protocol modelling Network performance Traffic engineering Signalling protocol BISDN

\section{INTRODUCTION}

The introduction of the asynchronous transfer mode (ATM) will allow BISDN to support a large variety of services. Advanced multimedia and multiparty applications, such as HDTV distribution and video conferencing, are expected to constitute an important part of the applications in BISDN. The RACE II project 'Multiservice Applications Governing Integrated Control' (MAGIC) ${ }^{1}$ aims at investigating the impact of advanced multiparty multimedia services on the signalling and the call handling of broadband telecommunication networks. It targets towards the design of the BISDN release $2 /$ 3 signalling protocols. As signalling is one of the key elements in the provision of services by modern telecommunication systems, the signalling system is essential in determining the overall performance of telecommunication services. Therefore the performance evaluation of signalling systems is recognized as an important issue at the early design stage of signalling protocols.
Owing to the complexity of signalling systems, signalling protocols have been designed in a multilayered architecture. Performance evaluation of multilayered protocol architectures has been a subject of study. ${ }^{2}$ For example, in Reference 3 an analytical model is presented for the OSI communication architecture based on the formal protocol specification using a finite state machine (FSM).

In Reference 4 a generic queueing network model is constructed for the ISDN Signalling System No. 7 (SS7) that is based directly on the structure and specifications of the protocol. For the solution of the model, an iterative decomposition algorithm is formulated. In this model, the network is considered as a whole. It is assumed that at each node in the network there are a number of entities in each layer that are interconnected through service access points (SAPs). Nodes at different geographical locations are interconnected through the entities of the physical layer. The entities at the top layer are assumed to be fed by sources. At the SAPs between adjacent entities, there are interface flow control queues. 
Each entity may carry out a number of functions as defined by the protocol specifications. Associated with each source is a closed routeing chain that follows a path through the entities of the network and the processing queues. Processing overheads are taken into account by assuming that whenever an entity processes a PDU, the PDU visits the set of processing queues. The set of processing queues may be shared by various layers. In addition, different priorities may also be assigned to the processing requests from different layers. The advantages of this methodology are as follows: it allows one to analyse large and complex networks in a relatively efficient manner; it takes into account the coupling that exists between layers both due to the interlayer traffic flows and the sharing of processing capacity. The main shortcoming is that it is difficult to ascertain the accuracy and the convergence properties of the iterative decomposition algorithm.

In Reference 5 the decomposition and aggregation technique is applied in the performance evaluation of SS7 as well. A queueing submodel is constructed for each layer. The submodel takes into account the various functions that may be carried out in the layer. Corresponding to each submodel are inputs and outputs for the PDUs that are passed between the layers. The inputs to the highest layer are connected to the traffic sources, and the outputs from the highest layer are connected to the traffic sinks. There are also sources which model end-user application process response times. Like the approach in Reference 4 it takes into account the coupling that exists between layers through the traffic flows. The possible weakness of the method is that it does not take into account the interactions between layers from the standpoint of processing.

In this paper we introduce a methodological approach to the modelling of BISDN signalling protocols being designed in the MAGIC project. Our approach is similar to that proposed in Reference 5. However, the difference lies in the fact that we do not intend to restrict the protocol modelling in order to obtain an analytically solvable model, instead we try to make a compromise between the accuracy and complexity of the model, such that the protocol model reflects the reality as much as possible, and is not too complicated to be solved.

Because the signalling system is so complicated, we believe that it is important to determine first the performance measures in which we are interested and then develop the system model. This will enable us to eliminate the irrelevant details, simplify the system model and speed up the solving process. For this reason, in Section 2 we address issues related to the definition of network performance parameters. In Section 3 the signalling system is decomposed into three submodels, i.e. the workload model, the signalling protocol model and the signalling transfer network model. Conclusions are given in Section 4.

\section{DEFINING NETWORK PERFORMANCE PARAMETERS}

Network performance is defined in the CCITT Recommendation E. $800^{6}$ as the ability of a network or network portion to provide the functions related to communications between users. It is defined in terms of parameters which are meaningful to the network ope rator and are used for the purpose of system design, configuration, operation and maintenance. It must be defined independently of terminal performance and user actions.

The principles of defining network performance parameters are suggested by CCITT. ${ }^{7}$ These principles led to the network performance objectives for connection processing delays in an ISDN. ${ }^{8}$ Network performance parameters such as overall connection set-up delay and alerting delay are defined. However, little work has been done in defining performance parameters for BISDN. Anagnostou et al. have extended the CCITT principles in defining quality of service (QoS) requirements in BISDN.9 ${ }^{9}$ RACE project 1082, 'QOSMIC' has made an attempt to study the definition of QoS in integrated broadband communications and the relation between QoS and network performance. ${ }^{10}$ However, as far as the network performance of signalling is concerned, none of them has taken into account the new features of BISDN, such as the multiparty multimedia aspects of the BISDN services and the separation of call and bearer control in the BISDN signalling.

BISDN is expected to provide advanced multiparty multimedia services. Calls offering the BISDN services can be dynamically modified during their lifetime. Unlike the traditional confirmed service requests such as those defined in the OSI standards (see Figure 1(a)) confirmed service requests in BISDN calls need a two-phase confirmation ${ }^{11.12}$ in order to perform negotiation among multiple parties (see Figure 1(b)). Called users are polled in the first phase. The network determines the outcome of the negotiation based on the responses from called users and its own status. Once the outcome is determined, the processing of the confirmed service request enters the second phase to commit or rollback the service request.

Taking into account this new feature of the BISDN services, a new device is needed to describe the relationship between message exchange events at each user's UNI so that network performance parameters can be defined based on events or states observable at the UNI. For this purpose we adopt a message flow model described in Section 2.1.

In multiparty multimedia calls supported by BISDN, the one-to-one mapping between a call and its underlying connection does not hold anymore. The separation of call and bearer control makes the sequential set-up of calls and connections possible. ${ }^{13}$ When a call set-up is acknowledged the connections of this call may not be available yet. Thus the call set-up delay alone does not say everything about 


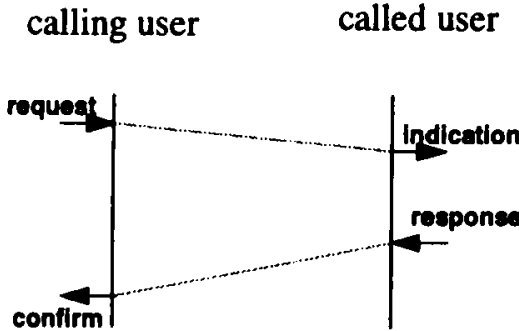

(a) two-party communication

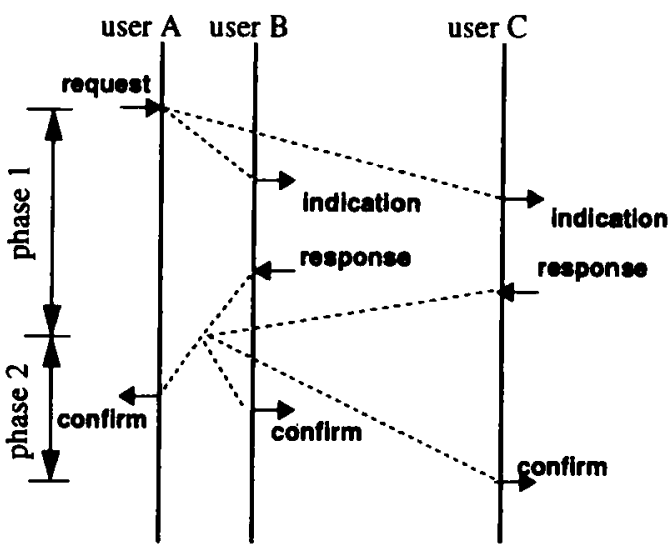

(b) multiparty communication

Figure 1. Confirmed service request

the speed of the set-up process, leading to a need for new performance parameters. In the MAGIC project several network performance parameters are considered. Definitions of these parameters are presented in Section 2.2.

\subsection{Message flow model}

A message flow model consists of a set of nodes, a set of edges and a bar. Construction of a message flow model starts from the node, also called the root, representing the initial requesting user. The initial requesting user, analogous to an OSI calling user, sends a request to the network which results in the delivery of other messages to one or more users. This is modelled by a node-to-multinode edge originating from the root node and ending at the nodes representing users who have received a message from the network. The users receiving a message from the network can be intermediate or final called users.

Introducing an intermediate called user enhances the flexibility in the sense that it allows a user to structure a call in which he does not participate, e.g. the user terminal equipment simply forwards calls to other access points. Upon the receipt of a message from the network, an intermediate called user will send a further request encapsulated in its response to the network. The request will in turn cause a message to be delivered to one or more users. This is modelled in the same manner as the request issued by the initial requesting user.

Final called users send only one response to the network. This is modelled by a node-to-bar edge originating from the node representing the final called user and ending at the bar. Once the outcome of the multiparty negotiation is determined by the network, bar-to-node edges are introduced in order to model the commitment or rollback of the service request to all the users. Ending nodes of bar-tonode edges are called leaves.

Let us give an example to illustrate how to construct the message flow model of a call set-up pro- cedure (Figure 2). Assume user $A$ wants to set-up a voice conference call with user $B$ and $C$. $A$ sends a message ( $m s g 1)$ to the network, which will cause a message to be delivered to $B$ and $C$ respectively at their own UNI. $C$ accepts the call by sending a response message ( $m s g 2)$ to the network. $B$ accepts the call and furthermore wants to ask $A$ to upgrade the call to a video conference, so he sends a message ( $m s g 3$ ) to the network. The message $m s g 3$ will on the one hand tell the network the acceptance of $B$, and on the other hand cause a message to be delivered to $A$ in order to inform $A$ that $B$ wants to set up a video connection. $A$ agrees with the request from $B$ and sends a response to the network ( $\mathrm{msg} 4$ ). Now that all parties have agreed with the call setup, the network will send a message to each of the users in the call to commit the call set-up. The call is a mixed voice/video conference call: all the users can hear each other, but only $A$ and $B$ can see each other through the video connection.

\subsection{Definition of network performance parameters}

(i) Call set-up delay

Definition

The call set-up delay is defined as the time duration from the instant when the initial requesting

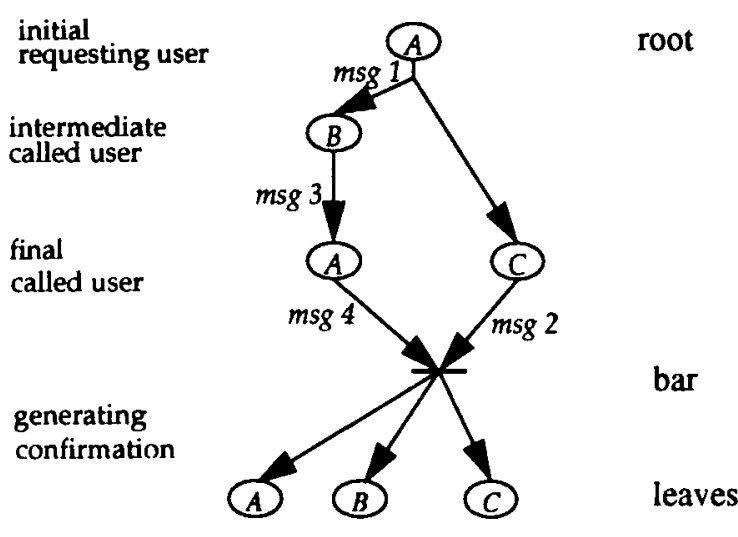

Figure 2. Message flow model of call set-up: an example 
user starts the call set-up procedure by sending a message to the network, until the call set-up procedure is committed or rolled back to the initial requesting user as well as all the intermediate and final called users, excluding the user response time(s).

\section{(ii) Bearer set-up delay}

\section{Definition}

The bearer set-up delay is defined as the time duration from the instant when the network starts the bearer set-up due to the receipt of a bearer setup request message from a user or from another network element until the requested bearer* is ready for use, excluding user response time(s) if any.

\section{(iii) Call blocking probability}

\section{Definition}

The call blocking probability is the probability of a call being denied due to the lack of network and/ or signalling resources to support the call.

\section{(iv) Bearer blocking probability}

\section{Definition}

The bearer blocking probability is defined as the probability of a bearer set-up transaction being rolled back because of the lack of resources, given that the call has been committed.

\section{(v) Release delay}

\section{Definition}

The release delay is defined as the time duration from the instant when a bearer release request is issued to the network until the concerned network resources are released.

The release delay is defined only for bearer release because it has effects on the utilization of the network resources. Release of a call or part of it is mostly a user-user signalling concern and therefore is not addressed here.

Call and bearer blocking probability are determined by the resource reservation and allocation strategy, and depend on the amount of network resources available in the network and needed for the call. They are determined by the signalling protocol through the consumption of the signalling resources. They do not depend on the terminal compatibility nor on the user actions.

Both call set-up delay and bearer set-up delay are determined by the signalling system including the

\footnotetext{
- The term 'bearer' in this paper means any physical resources needed for user information transmission. Examples are ATM virtual channel connection, conferencing bridges etc.
}

signalling transfer network, the reference configuration of the signalling network, the capacity of the processor(s) on which the signalling protocol runs and the complexity of the call. They also depend on the load of the signalling network, and they are not determined by the terminal performance nor by the user actions. These parameters must be understood in a different way than in Reference 9, in particular with respect to the exclusion of the user response times.

In defining the network performance parameters, those factors which do not depend on the network itself must be excluded. For example, when we talk about call blocking probability of the network, we do not consider the call set-up failure caused by the absence of the called user. In the definition of overall processing delay of a confirmed service request, the user-response times must be excluded. However, in multiparty communication, it is difficult to distinguish user-response times from network processing time.

Let us elaborate on the previous example. Figure 3 shows the temporal order of events at the UNIs of concerned users. The dashed lines in the Figure show the relation between events at the different UNIs and the dotted lines represent user response times. We can clearly see that some of the user-response times are overlapping with the system processing time, but we cannot simply subtract them from the overall call set-up delay.

The message flow model introduced in the previous section allows us to exclude user-response times systematically. In order to do so, the diagram of the message flow model is converted into a weighted directed graph in three steps. First, each node-to-multinode edge in the message flow model is replaced by several node-to-node edges. As an example, the message flow model in Figure 2 is converted into a directed diagram in Figure 4(a). Secondly, edges are assigned a weight, and message names and the users' names are removed from the diagram of the message flow model (see Figure 4(b)). The weight of an edge equals the total delay of a message, which includes processing and transmission. Processing delays are derived from the signalling protocol model (see Section 3.2), whereas transmission delays are extracted from the signalling transfer network model (Section 3.3). A node-tonode edge means that the user at the origin of the edge sends a message to the network, and this message causes the user at the end of the edge to receive a message from the network. This edge has a weight equal to the time duration from the instant when the originating user sends the message to the network until the destination user has received the message. A node-to-bar edge means that a response is sent to the network. The network represented by the bar can only start processing as soon as it has received all the responses. Thus a weight of zero is assigned to all the node-to-bar edges. The processing time of responses will be taken into account by the 


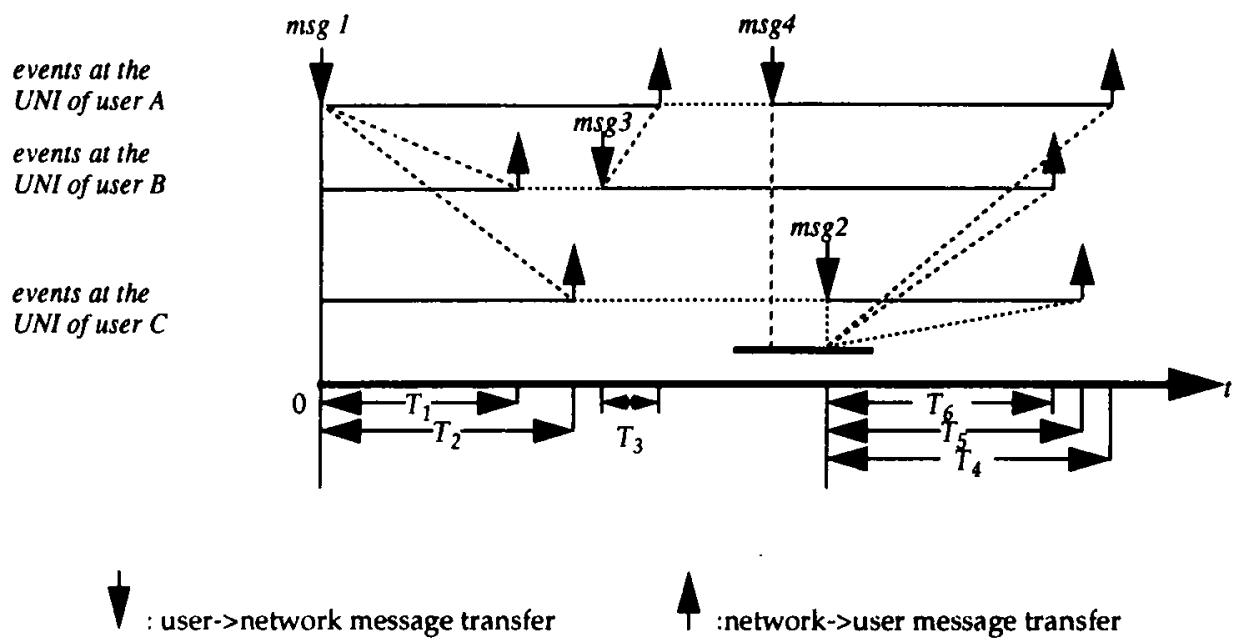

Figure 3. Temporal order of events at the UNIs of concerned users

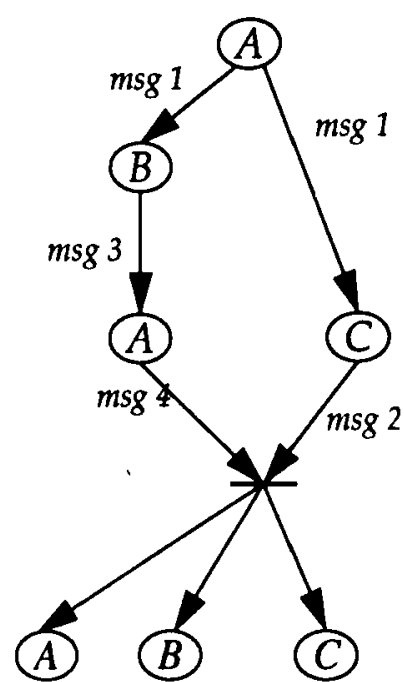

(a)

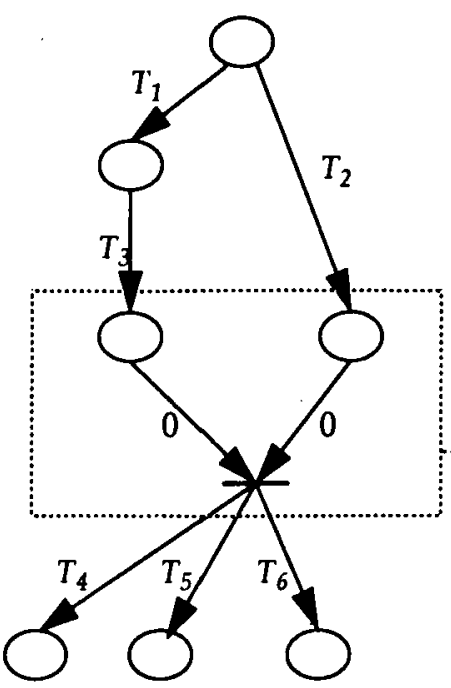

(b)

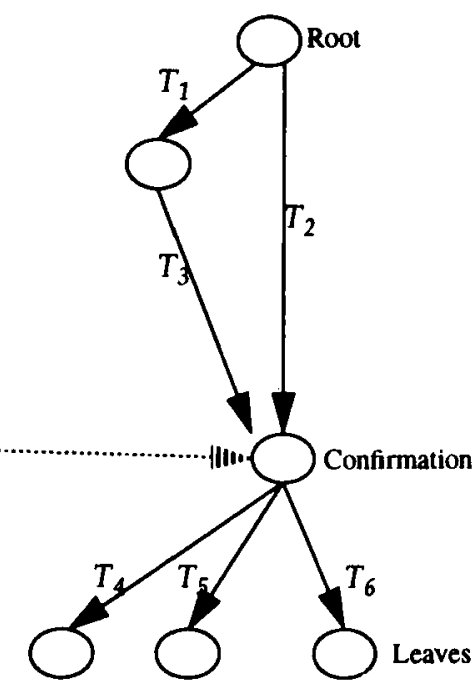

(c)

Figure 4. Convet message flow model into a weighted directed graph

weights of bar-to-node edges. A bar-to-node edge means that the network sends a confirmation to the user represented by the node at the end of the edge. It gets a weight equal to the time duration from the instant when the network has received the last response until the ending user receives the confirmation. The weights of edges are shown in Figure 3.

If we define the weight of a path as the sum of weights of all the edges along the path, we are able to obtain the overall processing delay of a confirmed service request excluding user-response times as the maximum of the weights of all possible paths from the root to a leaf node. For the example, the overall call set-up delay is as follows:

$$
\begin{aligned}
& \text { Call set-up delay = } \max \left(\begin{array}{l}
T_{1}+\mathrm{T}_{3}+T_{4}, T_{1}+T_{3}+T_{5}, \\
\\
T_{1}+T_{3}+T_{6}, T_{2}+T_{4}, \\
\left.T_{2}+T_{5}, T_{2}+T_{6}\right)
\end{array}\right.
\end{aligned}
$$

For a complex confirmed service request the help of graph theory is needed to calculate the processing delay. Thus we finally convert the message flow model into a weighted directed graph by removing the bar and all the node-to-bar edges from the diagram. Originating nodes of all the node-to-bar edges are merged into one node, called the confirmation node, which will replace the bar as the origin of all the bar-to-node edges, see Figure $4(\mathrm{c})$. This final step is allowed because we are interested in the weights of paths only. Now, we are able to use algorithms in graph theory to find the longest path from the root to the confirmation node. The overall processing delay excluding user-response times will then be the weight of this path plus the weight of the longest edge between the confirmation node and leaves. An algorithm to find the longest path is given, for example, in Section 2.6 of Reference 14 .

\section{MODELLING METHODOLOGY}

Signalling protocols, currently designed in the framework of the MAGIC project, follow the OSI 
application layer structure (ALS); see Figure 5. (For OSI/ALS structured BISDN signalling architecture, readers are referred to Reference 15). These are invoked by application processes in order to set up calls, allocate resources etc. Application processes are called the users of the signalling protocols in which we can identify functional entities each one performing a particular function. Functional entities may be geographically distributed, and the communication between them is supported by a signalling transfer network. In the first step of decomposition, the signalling system is divided into three submodels, namely the workload model, the signalling protocol model and the signalling transfer network model, as shown schematically in Figure 6.

The workload model is concerned with the quantitative aspects of the user behaviour as far as signalling is concerned. It describes the time and location behaviour of requests for calls and requests for the

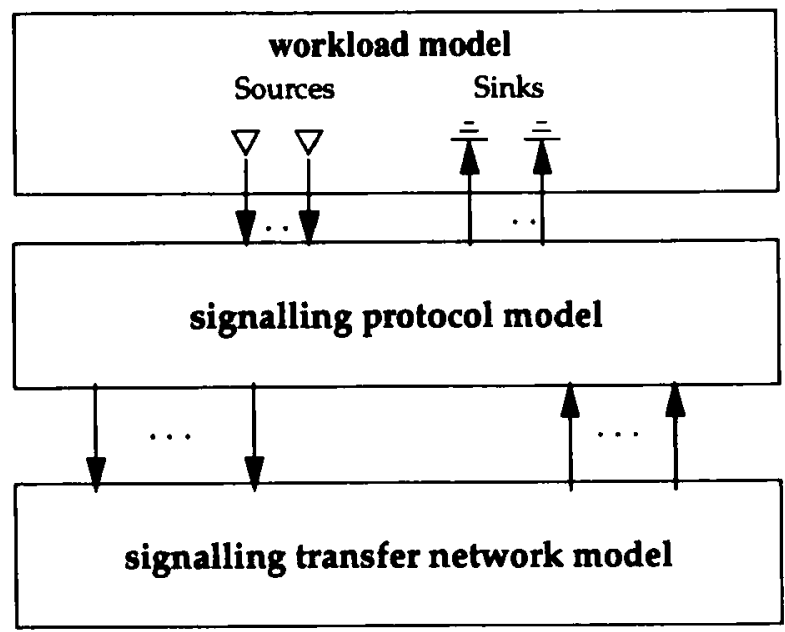

Figure 6. Decomposition of the signalling system model
TE

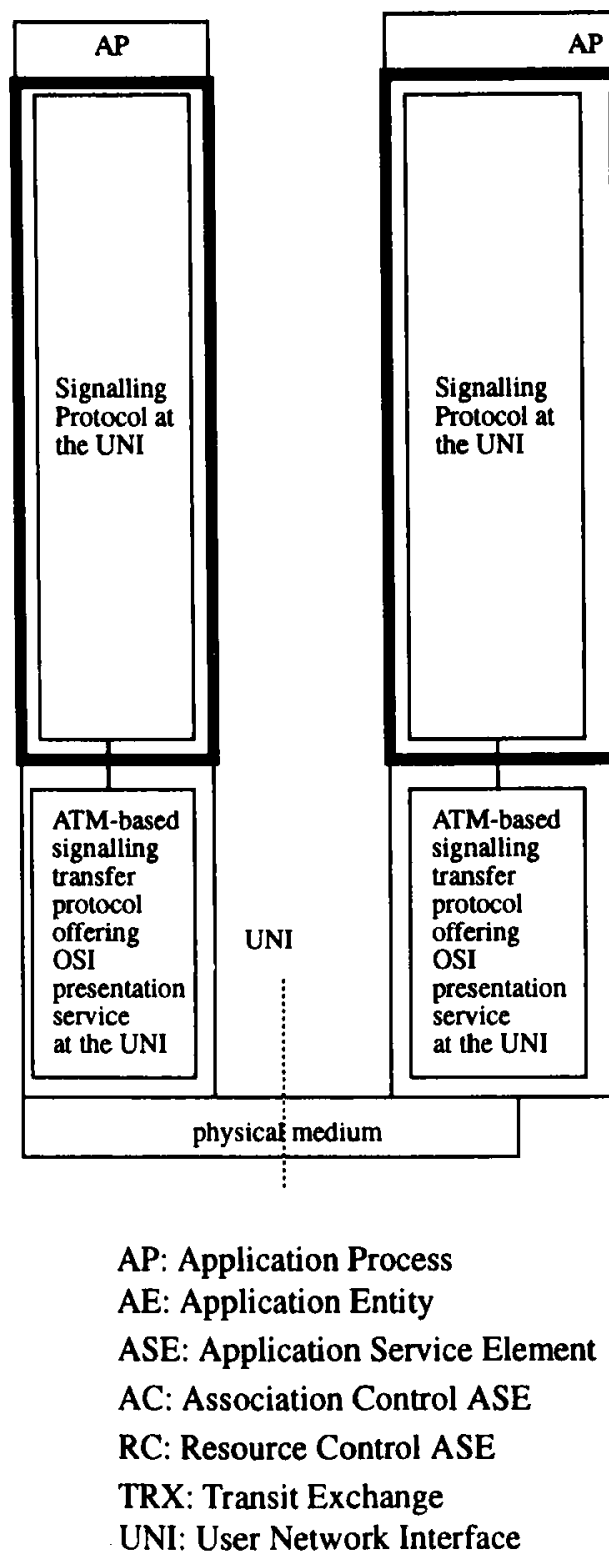

TRX

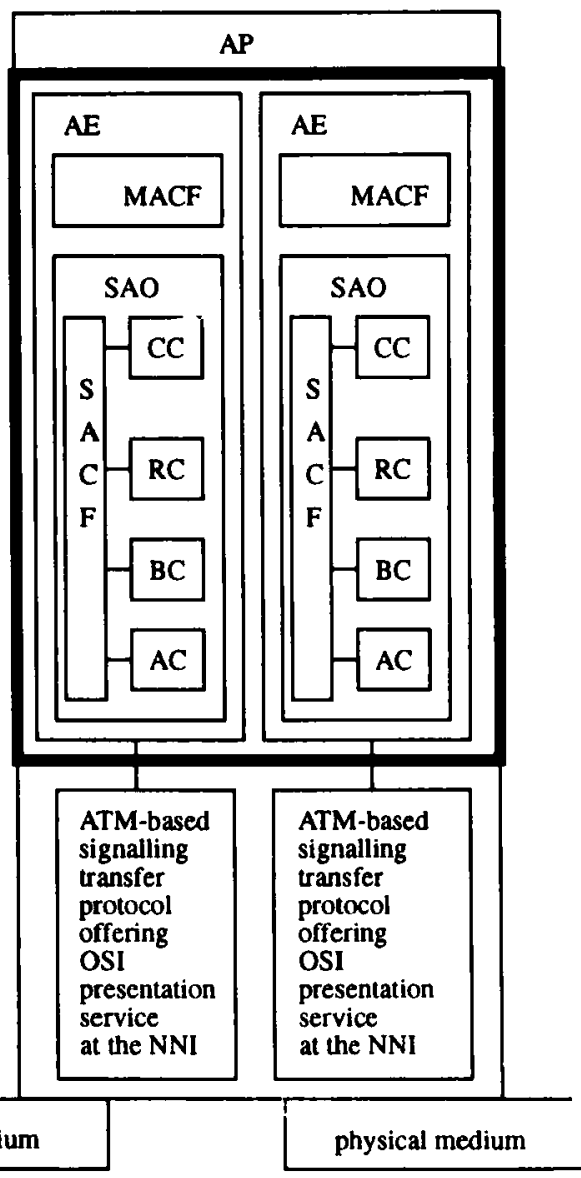

MACF: Multiple Association Control Function SACF: Single Association Control Function

SAO: Single Association Object

CC: Call Control ASE BC: Bearer Control ASE

TE: Terminal Equipment

LEX: Local EXchange

NNI: Network Node Interface

Figure 5. BISDN signalling protocol architecture 
modification of calls, e.g. by means of statistical and stochastic techniques.

The signalling protocol model is concerned with the modelling of the BISDN signalling protocols, studied in the MAGIC project. ${ }^{16}$ It also incorporates the modelling of the computing resources needed to support the execution of the signalling protocols. The signalling protocols are structured according to the OSI/ALS, and thus the signalling protocol model should present the abstraction of all the application service elements (ASE) performing signalling functions and describe the mutual interaction between ASEs. These ASEs include the call control ASE (CC), the resource control ASE (RC) and the bearer control ASE (BC).

The signalling transfer network model is developed as well. It models the transmission of signalling messages among signalling end points and signalling relays. The level of detail of the signalling transfer network modelling is such that the model is suitable for the performance evaluation of the signalling protocols.

\subsection{Workload model}

In principle, a signalling workload model should be able to accurately describe the user requests for new calls and for modifications of the parameters of existing calls. Since at the present time it is not possible to know the actual workload of the future BISDN, the model should be general enough to accommodate any scenario with respect to the number of potential users and their 'signalling' activity. A high-level view of the workload model used in our studies appears in Figure 7.

We distinguish two parts, each one further decomposed into a number of levels: the first part is the signalling protocol independent part (SPIP) and includes only user originated signalling activities, e.g. requests for new calls, modifications and release of existing calls as desired by the user. A call release initiated by the network does not belong to SPIP. The second part, termed the signalling protocol dependent part (SPDP) uses, as input, the stream of user requests coming from the upper SPIP and translates them to signalling messages (e.g. call and bearer control messages, messages relate to basic operations within the network such as bandwidth allocation and connection establishment etc.). The SPDP will be modelled in the following sections dealing with signalling protocol and transfer network modelling.

For analysing SPIP, we adopt a multilevel approach which recognizes the three levels of Figure 7 , namely the user level (UL), the service level (SL) and the modification request level (MRL). The novel idea of workload modelling as depicted in Figure 7 is that the model is user descriptive in the sense that the SPIP does not represent the direct input required for evaluating the performance of a layer protocol (see, for example, Reference 17). Instead, this traffic is created as a result of a vertical communication between adjacent levels according to specific rules reflecting the potential of a particular customer to use a service and to ask for modifications of this service during the lifetime of a call. We thus can identify the actual implications of user signalling activity to the signalling traffic without resorting to approximations and restrictive assumptions to guess how many users and how much activity a certain signalling traffic stream represents.

The capability of a user to modify the parameters of a service throughout the duration of a call is modelled in the MRL. Three categories of modification requests, whose combination can result in the highest possible number of signalling messages in the SPDP, are considered. The 'QoS' modifications refer to requests for degrading or upgrading the quality of a call in terms of allocated resources. The 'connection' modifications include requests for

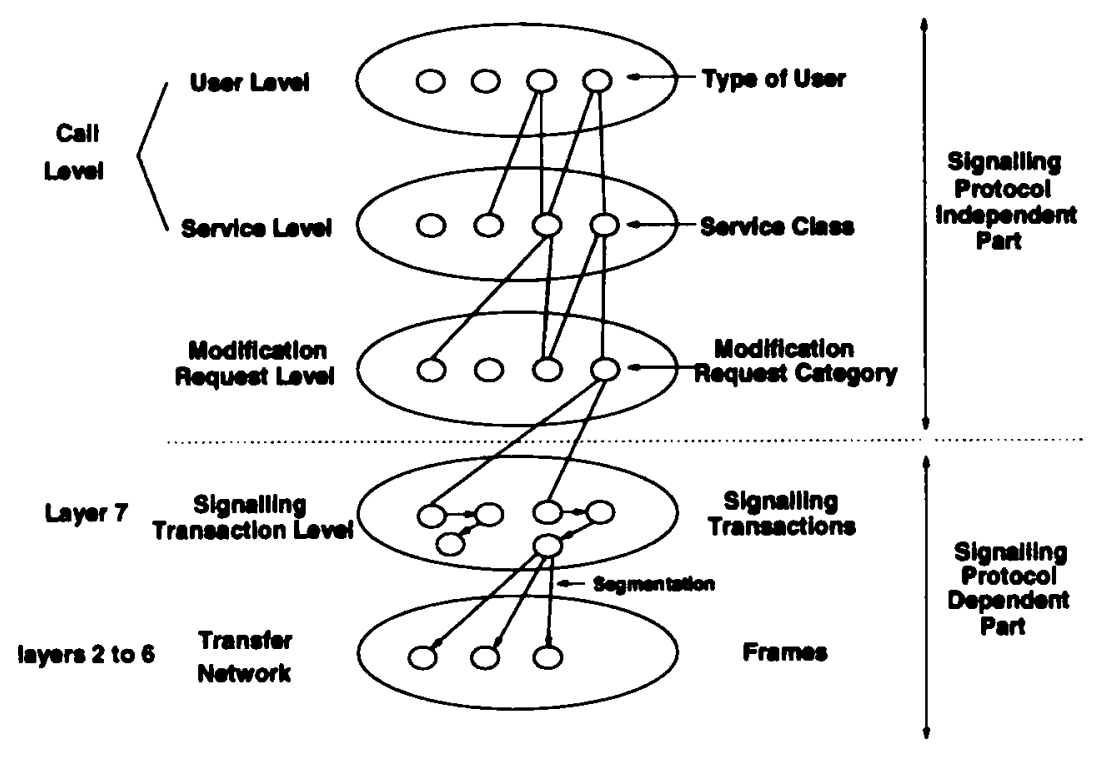

Figure 7. High-level workload modelling 
adding or removing a partner or a service component from an already established call, as well as merging or splitting calls. These modifications, if accepted, cause the creation or deletion of one or more connections between two or more users. The 'selection' modifications include requests for the selection of one among several 'channels' of a distribution service. A typical example is the selection of a TV channel.

The type and rate of user requests including call set-up and release, as well as modifications, depend on the particular service. This has led us to classifying services, using as a criterion the type of their modification requirements. In the SL of Figure 7 all services within one class have the same behaviour in terms of request rate for a particular user. Table I illustrates a possible service classification according to the modification categories of the MRL giving some typical service examples.

The type of service and the modification request rate depend on the particular user type. In our study we consider three wide types of users, namely residential, business and mobile users. We can then proceed with scenarios reflecting the expected number of user types in a specific area. Paradigms of user distributions are reported in, for example, Reference 18.

The above classification is a useful framework for defining workload scenarios that take into account both the number and type of users, as well as the required services. To do so, we define a set of traffic load parameters for each level of Figure 7 and perform a synthesis of them according to their physical relations, resulting in the three-level traffic load model (Figure 8). The call level (CAL) consists of the user requests for services and is described by the statistical parameters residing in the UL and SL of Figure 7. These are listed in Table II for the user types and service classes defined earlier in this section. The modification request per call level (MCR) is described by the statistical parameters of the MRL of Figure 7. Each call of the CAL represents a number of request messages required for the set-up of the call, all the modifications during the call and the release of the call. The expected number of modification messages of a particular modification category depends on the service class and on the type of the user originating the modification request, as well as on the duration of the

Table I. Service classes and modifications

\begin{tabular}{lcccc}
\hline Class & $\begin{array}{c}\text { Example } \\
\text { of services }\end{array}$ & QoS & Connection Selection \\
\hline 1 & $\begin{array}{c}\text { telephony } \\
\text { file transfer }\end{array}$ & $\mathrm{X}$ & & \\
2 & video-telephony & & $\mathrm{X}$ & $\mathrm{X}$ \\
3 & video on demand & & & $\mathrm{X}$ \\
4 & TV distribution & $\mathrm{X}$ & $\mathrm{X}$ & \\
\hline 6 & video-conference & $\mathrm{X}$ & $\mathrm{X}$ & \\
\hline
\end{tabular}

call. The duration of the call in turn depends on the type of user and the service class. The related parameters are listed in Table III.

Based on the statistics of the CAL and MCR levels which are actually matched to physical quantities, we can obtain the multiplexed stream of signalling transactions. This will be in turn translated into signalling messages by the SPDP model according to the adopted signalling protocol stack. It should be noted that workload modelling as performed in the SPIP includes the user signalling activity as enforced by another user or the network in a statistical manner. Alternatively, the enforced user signalling activity can be modelled in the SPDP by injecting the corresponding protocol signalling messages in the signalling traffic generated by the signalling protocol model. The reason is that the enforced signalling messages are not necessarily a result of user signalling activity but rather a reaction of the underlying signalling protocol to a received signalling message.

Depending on the level of detail to which the user signalling activity is represented, we can either apply analytical or simulation methods to obtain the multiplexed stream of signalling transcations. Analytical solutions imply that the user signalling activity for a particular service follows a known distribution. The same holds for the duration of a call of a particular service class and for a specific user type. For example, if we assume that the duration of a call is exponentially distributed, $a_{i j}$ is the mean value of a Poisson distribution describing the arrivals of calls of a specific service class $i$ from a specific user type $j$, and the modification rate within a call follows also Poisson distribution with a mean value $m_{i j}=\left[\mathrm{QoS}_{i j}+\operatorname{conn}_{i j}+\operatorname{sel}_{i j}\right]$, the stream of user $j$ transactions for $n$ calls of service $i$ is described by a Markov modulated Poisson process (MMPP) with an instantaneous transactions arrival rate given by $a_{i j n}=m_{i j} n$. In the most general case, however, the distributions will not be known and their synthesis would not be amenable to analytical description. For these cases closer to the real situation, the model can produce the required user transaction distributions through simulation experiments. A similar approach has been followed in Reference 18.

\subsection{Signalling protocol model}

Figure 9 presents an overview of the global functional modelling of the MAGIC signalling protocols. It consists of three parts: the control part including control functional entities and objects desribing logical network resources, the call model providing a local view of the status of a call, and the user plane model (UPM) which is an abstract representation of the physical resources managed by the network management.

In the control part, $\mathrm{CC}$ entities are concerned with negotiating telecommunication service, $\mathrm{BC}$ 


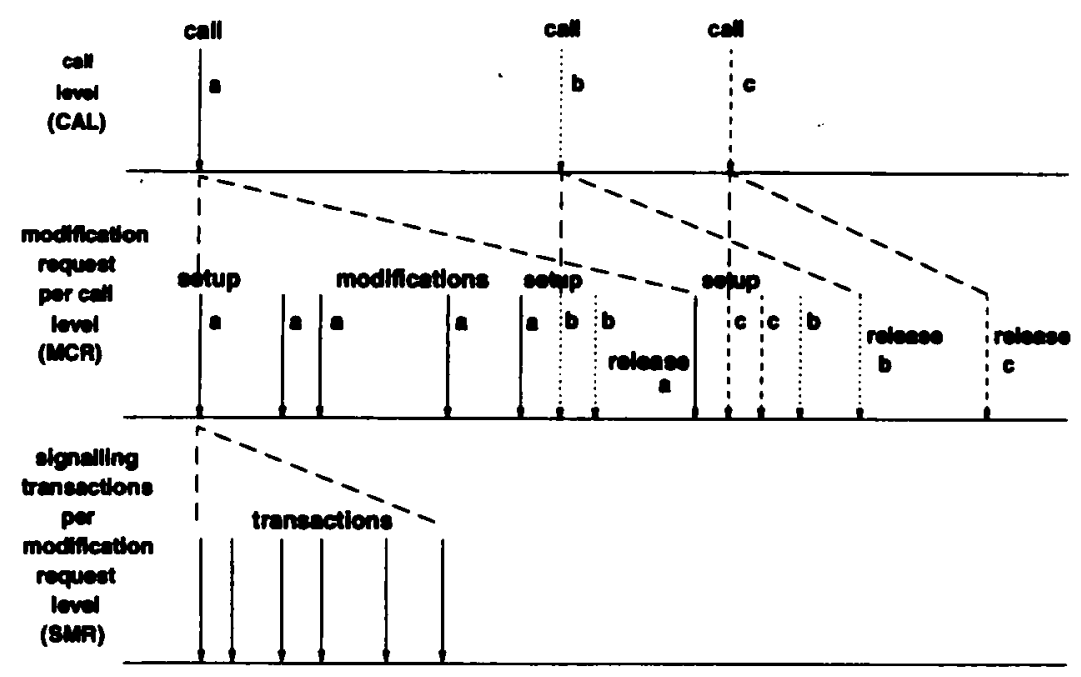

Figure 8. The three levels of workload modelling

Table II. Parameters at the CAL level

\begin{tabular}{lc}
\hline$a_{i j}$ & Call rate of $i$-class services, $j$-type users \\
$u_{j}$ & total call rate of $j$-type users, $u_{j}=\sum_{i} a_{i j}$ \\
$s_{i}$ & total call rate of $i$-class services, $s_{i}=\sum_{j} a_{i j}$ \\
$a$ & total call rate, $a=\sum_{i} s_{i}=\sum_{j} u_{j}=\sum_{i, j} a_{i j}$ \\
\hline
\end{tabular}

entities are concerned with bearer resources on a link-by-link basis, and RC entities* configure, allocate and control special resources that are internal to the network.

A user requests telecommunication services from the $\mathrm{CC}$ entity. Upon receiving a service request expressed by means of operations on the objects of the call model, the $\mathrm{CC}$ entity changes the local view of the user by creating, modifying and deleting the objects of the call model. It also communicates with peer $\mathrm{CC}$ entities to instruct them to change their

Table III. Parameters at the MCR level

\begin{tabular}{ll}
$\operatorname{QoS}_{i j}$ & $\begin{array}{l}\text { QoS modification rate during the lifetime of a } \\
\text { call, of } i \text {-class services, } j \text {-type users } \\
\text { conn }_{i j}\end{array}$ \\
$\begin{array}{l}\text { Connection modification rate during the } \\
\text { lifetime of a call, of } i \text {-class services, } j \text {-type } \\
\text { users }\end{array}$ \\
sel $_{i j}$ & $\begin{array}{l}\text { Selection modification rate during the lifetime } \\
\text { of a call, of } i \text {-class services, } j \text {-type users } \\
\text { Duration of a call, of } i \text {-class services, } j \text {-type } \\
\text { users }\end{array}$ \\
\hline
\end{tabular}

\footnotetext{
* The introduction of RC in the MAGIC stage 2 functional model allows separated control of special resources inside the network. It gives the network greater freedom in its distribution and use of special resources. For details on the MAGIC stage 2 functional model, readers are referred to Reference 19.
}

local view of the call. The CC entity will, sooner or later, invoke the RC entity to implement the call physically. This requires the translation from the objects of the abstract call model to the RC model objects, which represent the logical resources needed to implement the service request.

The RC entity makes use of the services of the $\mathrm{BC}$ level to set-up, modify and release ATM virtual channel connections by translating those $\mathrm{RC}$ model objects representing connections into $\mathrm{BC}$ model objects such as source, destination, link capacity etc. The RC entity creates UPM objects implementing those which are to be allocated by special resources. If the special resources are not availble locally, the RC entity finds out the location and communicates with the corresponding RC entity to demand allocation of the special resources.

The BC entity creates objects of UPM for the allocation of a virtual channel link, and communicates with peer BC entities so that end-to-end connections can be established link-by-link.

Creation of a UPM object will automatically generate a request to the network management entity for the allocation of resources, e.g. bandwidth. The UPM object is deleted when resources are deallocated.

Based on the global functional model, the signalling protocol model can be developed. Figure 10 shows the signalling protocol model for a signalling end point at the edge of the network, where all the $\mathrm{CC}, \mathrm{RC}$ and $\mathrm{BC}$ entities exist. For signalling end points within the network, those queues and related transit arcs modelling the functional entities not existing in these nodes can be removed.

The protocol model is basically a network of queues with extensions to specify synchronization and dependency. In the protocol model, the CC entity is modelled by two queues, one for local request/response processing $(\mathrm{CCr})$ and the other (CCc) for the processing of $\mathrm{CC}$ messages from the network, i.e. remote requests and responses. The 
Control Part

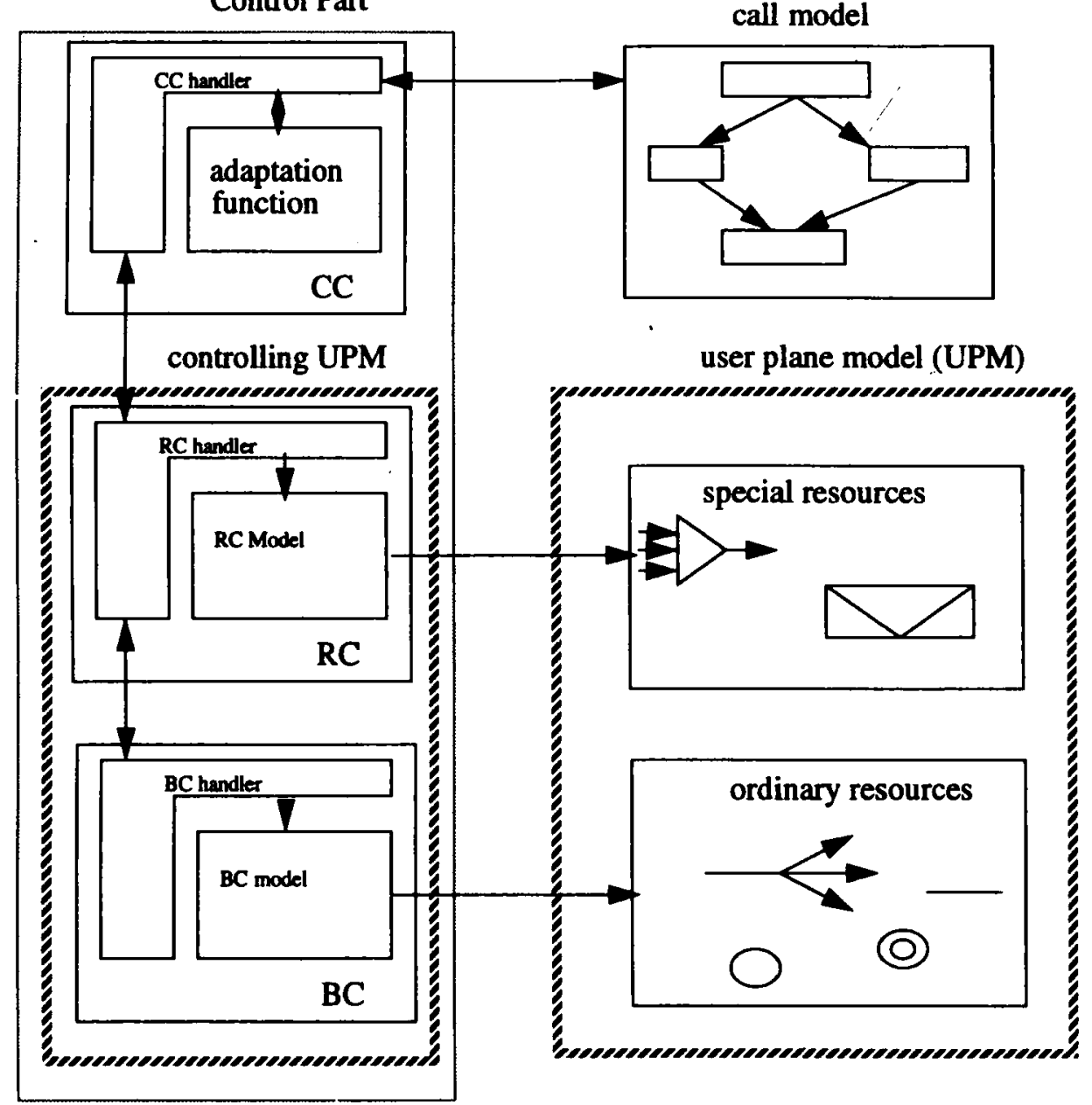

Figure 9. Global functional modelling

RC entity is also modelled by two queues: one $(\mathrm{RCr})$ models the processing of the resource request from local CC entity or from remote RC entities, and the other $(\mathrm{RCi})$ the processing of the responses from the network management as well as from remote $\mathrm{RC}$ and $\mathrm{BC}$ entities. The $\mathrm{BC}$ entity is modelled by a queue $(\mathrm{BCq})$ modelling the processing of connection requests from both $R C$ and peer $B C$ entities and the processing of responses from peer BC entities. Queue M1 and M2 model the network management processing for the allocation of special resources and set-up of VCLs, respectively.

Customers generated by the workload model are of two types: one models call or modification requests, the other models responses to incoming requests. Once generated, the customer enters the $\mathrm{CCr}$ queue. After being served in the $\mathrm{CCr}$ queue, with probability $\left(1-p_{0}\right)$ the service request is refused so the customer transits to the $\mathrm{CCc}$ generating a confirmation to the user. If the service request is accepted (with probability $p_{0}$ ), both or either of the following may happen: invoking communications with peer $\mathrm{CC}$ entities in other signalling end points; invoking the local RC entity to implement the service request physically. This is modelled by the duplication point following the $\mathrm{CCr}$ queue. With probability $p_{1}$ the customer is duplicated and transit to the signalling transfer network model in order to model the communication with a peer CC entity; with probability $p_{2}$ the customer is duplicated and transits to the $\mathrm{RCr}$ queue. The values of $p_{1}$ and $p_{2}$ depend on the protocol design option (either simultaneous or sequential set-up of calls and bearers), the type of customers (either a request or a response) and the type of services. A constraint is that $\left(1-p_{1}\right)\left(1-p_{2}\right)=0$, meaning that the customer will never disappear at the duplication point.

Customers entering $\mathrm{RCr}$ can either be duplications of customers leaving $\mathrm{CCr}$, or customers from a remote $\mathrm{RC}$ entity through the signalling transfer network. As mentioned before, the RC entity invokes the BC entity for the set-up of VCLs; concurrently, it may also invoke the network management (M1) or communicate with remote RC entities for the allocation of special resources. Thus a customer leaving the $\mathrm{RCr}$ queue is duplicated and transits to the $\mathrm{BC}$ queue with probability $p_{5}=1$, is duplicated and transits to the M1 queue with probability $p_{4}$, and to the signalling transfer network with probability $p_{3}$.

Customers of the $\mathrm{BCq}$ queue may come from a local RC entity modelled by the RCr or through a signalling transfer network moel from a remote $\mathrm{BC}$ entity. After being served in the $\mathrm{BCq}$ queue, the 


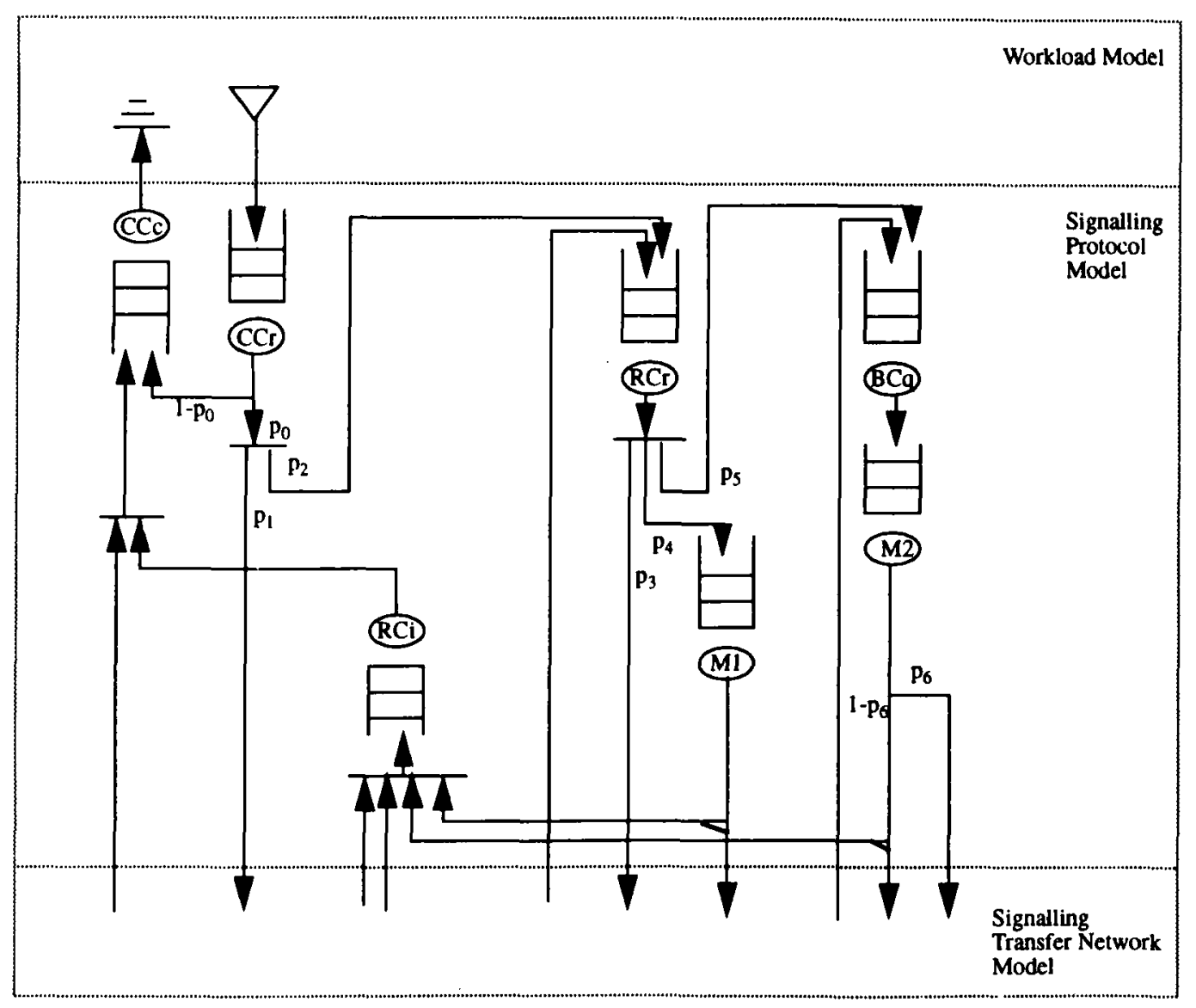

Figure 10. Signalling protocol model

customer transits to $\mathrm{M} 2$. With probability $p_{6}$, local resource allocation succeeds and the customer transits to the signalling transfer network model either to communicate with the peer $\mathrm{BC}$ entity for setting up the next link or to confirm the remote $\mathrm{RCi}$ that the requested link has been allocated. With probability $\left(1-p_{6}\right)$, local resource allocation fails. In this case, the customer transits either directly to the $\mathrm{RCi}$ queue if the request was from the local $\mathrm{RC}$ entity, or through the signalling transfer network to $\mathrm{RCi}$ if the request was from the peer $\mathrm{BC}$ entity. The dependency of the two possible transition routes is presented by a branch with a cross bar.

Customers leaving the M1 queue indicate, in reality, whether or not the special resources have been allocated. They transit either directly to the $\mathrm{RCi}$ queue if the customer was originally from the local RC entity; or through the signalling transfer network to the remote $\mathrm{RCi}$ in the signalling end point where the special resource request came from.

Because a confirmation of resource allocation can be made only when the responses from all the concerned $\mathrm{RC} / \mathrm{BC}$ entities have arrived, a combination point is placed at the entrance of the $\mathrm{RCi}$ queue. One customer can proceed to $\mathrm{RCi}$ only if all its duplications made after the service at $\mathrm{RCr}$ have arrived at this combination point.

For a similar reason, customers leaving $\mathrm{RCi}$ for $\mathrm{CCc}$ have to wait at the combination point at the entrance of the $\mathrm{CCc}$ queue for their duplications made after the service at the $\mathrm{CCr}$ queue.

A customer from the signalling transfer network towards the $\mathrm{CCc}$ queue can either be a request or response from a remote $\mathrm{CC}$ entity. $\mathrm{A}$ request from a remote $\mathrm{CC}$ entity has no duplication, and thus can proceed to $\mathrm{CCc}$ then to the workload model. If a response from a remote $\mathrm{CC}$ entity has duplications for setting up a multiparty call or for resource allocation, it has to wait until all its duplications arrive at the combination point. All the customers will be combined with the duplications to form one customer and proceed to the CCc queue.

\subsection{Signalling transfer network model}

The signalling transfer network deals with the transfer of the signalling information provided by the signalling applications (layer 7 entitites). The elements in the signalling transfer network are widely classified into signalling end points and signalling relays. The main difference between them is that, apart from the signalling information transfer capability provided by both, signalling end points can also interpret signalling information. This may be the global signalling information including call handling or the subset required for bearer control. From the signalling transfer network viewpoint only the protocols operating at the signalling relay part 


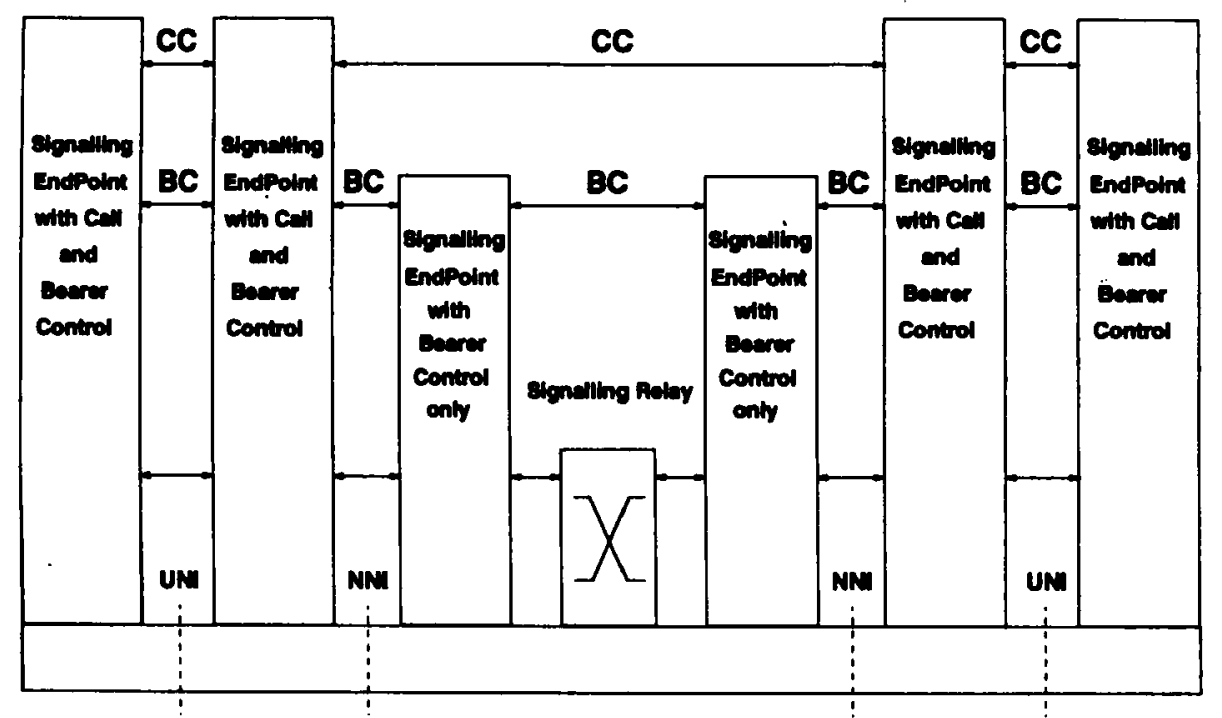

Figure 11. One typical network configuration

of any signalling element are essential factors for evaluating the performance. A typical configuration including all signalling elements is shown in Figure 11. Two options are currently considered regarding signalling information transfer in BISDN. ${ }^{20}$ The first one uses typical ATM switches/ cross-connects, whereas the second implements a layer 3 connectionless service over the ATM network. This option is only applicable at the network node interface (NNI) and the resulting protocol stack is illustrated in Figure 12. At the user network interface (UNI), the protocol stack is the same except for the layer 3 protocols (SCCP and MTP3) which are absent at the UNI.

In general each layer or functional group within a layer can be modelled as a queueing system with a generally distributed service time reflecting the processing time of the layer protocol. This appears in Figure 13. The processing time for each PDU varies according to the executed code length, the system clock rate and the particular CPU technology used. This allows processing speed requirements to

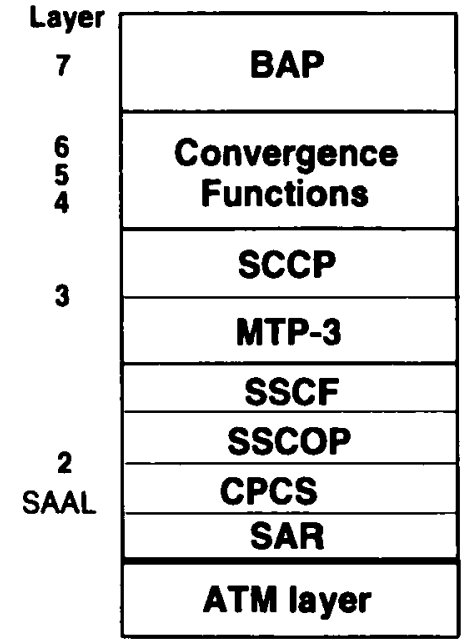

Figure 12. Protocol stack at the NNI

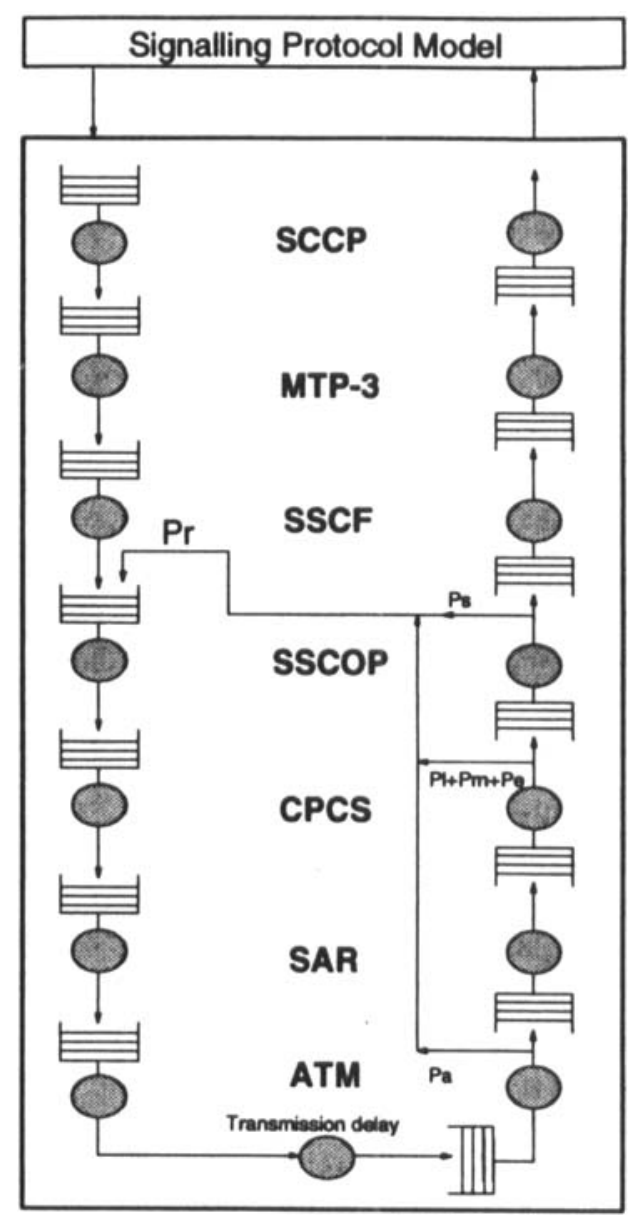

Figure 13. Transfer network model

be defined for all layer protocols from SCCP to SAR and possible performance bottlenecks to be identified stemming from the fact that these protocols will be based on software realizations. We can also identify the need for flow control mechanisms by observing buffer states at different layers and estimating both the queueing and processing delays experienced by PDUs. In order to generate the actual signalling traffic stream entering the ATM 
layer we take into account both the header overhead appending in the incoming SDUs at all layers of the protocol stack, as well as the number of retransmissions required for the erroneous PDUs. These, together with the processing delays, define the actual bandwidth consumed by signalling connections and constitute a useful framework for recommending on the portion of bandwidth required for the signalling activity of a service user.

The signalling connection control part (SCCP), as well as the message transfer part level 3 (MTP3) have been borrowed from SS7,21,22 suitably modified and adapted to the underlying ATM environment. The most important SCCP operation addressed so far for BISDN signalling is the global title addressing capability, ${ }^{20}$ and as such it is modelled in the service time of the corresponding queue (in Reference 23 this time is taken equal to $25 \mathrm{~ms}$ ). The MTP-3 protocol functionality is extensively used within BISDN, allowing the employment of the models developed for SS7 networks. ${ }^{4,18}$ The approach in Reference 4 is different from the one followed in this study in the sense that processing speed requirements and flow control links are now identified not in terms of data units delay but rather by specifying the signalling workload in the queue. To do so, a data unit entering the boundaries of the signalling message handling part (MHP) or MTP-3 follows one of the possible routes defined by combinations of the available functions with a certain probability. The profile of the stream exiting the MHP depends on these probabilities. ${ }^{4}$ Apart from MHP, SS7 recognizes an additional signalling network management (SNM) part. SNM is only activated when fault or congestion is experienced on a link. The SNM is not explicitly modelled, instead through different bandwidth allocation schemes for signalling traffic and different CPU speeds we evaluate the need for diverting traffic among links and exercise flow control in the signalling nodes.

SAAL is divided into three layers: the service specific convergence sublayer (SSCS), the common part convergence sublayer (CPCS) and the segmentation and reassembly sublayer (SAR); SSCS is further divided into the service specific co-ordination function (SSCF) and the service specific connection oriented peer-to-peer protocol (SSCOP) (Figure 12).

SSCF performs a co-ordination function between the service required by the SAAL user and the service provided by the SSCOP. This co-ordination function simply maps the SAAL primitives to SSCOP signals and vice versa. This sublayer can be modelled as a deterministic server, since incoming primitives are transparently translated into outgoing primitives. ${ }^{24}$ The functions of the SSCOP sublayer which affect the performance of the signalling transfer network are the retransmission of SSCOP-PDUs, the flow control and the SSCOP-PCI error detection. ${ }^{25}$ Retransmission of SSCOP-PDUs is performed if an error is detected by the lower CPCS sublayer protocol, if the number of received SSCOPPDUs exceeds the receiver window size and if an uncorrectable error is detected at the SSCOP-PCI. Retransmission affects performance in terms of additional signalling traffic and processing overhead. It is taken into account in the model of Figure 13 by inserting SSCOP-SDUs in the SSCOP queue with probability $p_{r}$, i.e. the probability of an uncorrectable error is identified in the CPCS or SSCOP sublayers. These may be the loss or misinsertion of an ATM cell conveying signalling information (with probabilities $p_{1}$ and $p_{m}$, respectively), an uncorrectable bit error in the ATM cell header $\left(p_{\mathrm{a}}\right)$ or in the ATM cell payload $\left(p_{\mathrm{e}}\right)$ or in the SSCOP-PCI $\left(p_{\mathrm{s}}\right)$.

The CPCS protocol contributes to the performance of the system only indirectly, i.e. it calls for retransmission of SSCOP-PDUs if either an uncorrectable error is detected by a four-octet CRC $\left(p_{\mathrm{e}}\right)$ or the reassembled PDU size does not comply to the two-octet length indicator value $\left(p_{1}+p_{\mathrm{m}}\right) .{ }^{25} \mathrm{Pad}$ ding is used so that the CPCS-PDU will always be a multiple of 48 octets. This affects performance in the way described below.

The SAR sublayer protocol segments SAR-SDUs into 48-octet blocks; no SAR-PCI is appended. Segmentation is always performed in sequential order so that multiplexing on a SAR-PDU level is justifiably excluded. SDUs of the same signalling connection are segmented in sequence and a VCI value is exclusively allocated to this signalling connection. With the above and given that sequence integrity is a built-in property of ATM, proper reassembly of SAR-PDUs is enabled at the receiver by virtue of the payload type subfield of the ATM cell header which indicates the last SAR-PDU of a SAR-SDU. The passage of the SAR-SDU across the SAR service boundary is modelled as a bulk arrival of cells. ${ }^{26}$ The number of cells in the bulk is given by the equation

$$
n=\frac{\mathrm{SAR}-\mathrm{SDU}}{48}
$$

Since the number of SAR-SDU octets is always an integral multiple of 48 (it is the CPCS responsibility to achieve this by appropriately filling the padding field), $n$ is an integer. The SAR-SDU size distribution in cells (48-octets) is discrete with a maximum depending on the PDU structure of the protocols residing above the SAR. An SCCP-SDU of say $x$ octets, will appear at the SAR sublayer service boundary as an SDU with $x^{\prime}$ octets size. The additional $\left(x^{\prime}-x\right)$ octets are the PCIs of the SCCP, MTP-3, SSCOP and CPCS sublayers as well as the padding field of the CPCS-PDU. We can assume that the SAR server is deterministic, with $\alpha$ being the service time per segment. The service time on a SAR-SDU basis becomes proportional to $n$.

In the ATM layer and below we are only concerned with the transmission and propagation delays 
depending on the available bandwidth for signalling information transfer as well as on the selected topology in terms of distance. The direct association of virtual channel connections to the signalling activity of a particularly call makes it flexible enough to derive statistics not only for signalling traffic in general but also for the specific service user. It should be noted that ATM-related performance issues, such as the use of the cell loss priority (CLP) bit for signalling cells, can be easily assessed through the set-up depicted in Figure 11 and for different background user information traffic. Also using a variance of this configuration, i.e. point-to-multipoint signalling configuration, we can properly dimension the bandwidth required for the default VC connection ( $\mathrm{VCI}=5$ ) dedicated to support signalling information transfer within the default VP connection (VPI $=0$ ).

\section{CONCLUSIONS}

We have presented a methodology for analysing the performance of complex BISDN signalling protocols supporting multiparty multimedia services. New network performance parameters have been introduced along with a message flow model towards the objective of deriving performance results associated with the special features of the BISDN signalling.

A flexible multi-level signalling system model has been developed. The model is easily decomposed into three parts, each one covering a different component of the overall signalling performance. The workload model incorporates the issue of user description as far as signalling activity is concerned and furthermore introduces an open classification of services according to the possible modifications they can suffer. The signalling protocol model is derived from the global functional model and information flows realized by an extended network of queues. The same approach is taken for the signalling transfer network. The overall model is amenable to analysis given that approximations and well known distributions are used. On the other hand the model is general enough to fit a variety of signalling protocols and configurations.

A lot of issues related to the BISDN signalling and protocol design can be resolved by applying the above model. Examples are the processing requirements of the signalling protocols, the bandwidth allocation for signalling connections of a high activity user etc. This was actually the intention for establishing a special task force on performance in the framework of the RACE II project MAGIC. ${ }^{1}$ The authors wish to express their gratitude to all partners involved in the project for their collaboration and valuable comments.

\section{REFERENCES}

1. C. M. W. Gabriël, J. J. M. Heffels, X. Hou and H. Ouibrahim, 'Heading towards an advanced signalling system for multimedia, multiparty services in broadband ISDN', Electronics \& Communication Engineering Journal, 5 (2), 103-112 (1993).

2. A. E. Conway, 'A perspective on the analytical performance evaluation of multilayered communication protocol architectures', IEEE J. Selected Areas in Communications, 9, (1), 4-14 (1991).

3. P. S. Kritzinger, 'A performance model of the OSI communication architecture', IEEE Trans. Communications, 36, (6), 554-563 (1986).

4. A. Conway, 'Queueing network modelling of signalling system No. 7', Proc. Globecom'90, San Diego, USA, December 1990.

5. G. Willmann and P. J. Kühn, 'Performance modelling of signalling system No. 7', IEEE Communications Magazine, July 1990 , pp. 44-56.

6. CCITT Recommendation E.800, 'Quality of service and dependability vocabulary', Blue Book, Melbourne, November 1988.

7. CCITT Recommendation 1.350 , 'General aspects of quality of service and network performance in digital networks, including ISDN', Blue Book, Melbourne, November 1988.

8. CCITT Recommendation 1.352, 'Network performance objectives for connection processing delays in an ISDN', Blue Book, Melbourne, November 1988.

9. M. E. Anagnostou, M. E. Theologou, K. M. Vlakos, D. Tournis and E. N. Protonotarios, 'Quality of service requirements in ATM-based B-ISDNs', Computer Communications, 14, (4), 197-204 (1991).

10. QOSMIC Consortium, QOSMIC Project Panorama, version 2, February 1993.

11. G. J. Heijenk, X. Hou and I. G. Niemegeers, 'Service description of communication systems supporting multimedia multi-user applications', Memoranda Informatica 93 34. Department of Computer Science, University of Twente, Enschede, The Netherlands, June 1993.

12. S. Minzer, 'A signalling protocol for complex multimedia services', IEEE Journal on Selected Areas in Communications, 9, (9), 1383-1394 (1991).

13. P.Blankers and $K$. Keskin, 'The separation between call control and connection control', in A. Casaca (ed.), Broadband Communications, Proceedings of the IFIP TC6 Workshop on Broadbank Communications, Estoril, Portugal, January 1992, pp. 125-134.

14. V. Chachra, P. M., Ghare and J. M. Moore, Applications of Graph Theory Algorithms, North-Holland New York, 1979.

15. A. Paglialunga and M. Siviero, 'ISCP (ISDN signalling control party): the best candidate for the target broadbandISDN signalling protocol', European Transactions on Telecommunications and Related Technologies, 4, (2), 193-200 (1993).

16. RACE II/MAGIC Deliverable 5, 'Signalling protocol for BISDN and interworking', June 1993.

17. A. Schmidt and R. Campbell, 'Internet protocol traffic analysis with applications for ATM switch design', $A C M$ SIGCOMM, Computer Communications Review, 23, (2), 39-52 (1993).

18. S. S. Liu, 'Impacts of signalling-intensive local services (SILS) on B-ISDN switching systems: a simulation study', Computer Networks and ISDN Systems, 25, 121-143 (1992).

19. RACE II/MAGIC Deliverable 1, 'Stage 2 functional model and information models', March 1993.

20. RACE II/MAGIC Deliverable 9, 'Signalling network architecture', September 1993.

21. D. R. Manfield, G. Millsteed and M. Zukerman, 'Congestion control in SS7 signaling networks', IEEE Communication Magazine, 31, (6), 50-57 (1993).

22. A. R. Modarressi and R. A. Skoog, 'Signalling system No. 7: a tutorial', IEEE Communication Magazine, 28, (7), 19-35 (1990).

23. R. A. Skoog and A. R. Modarressi, 'Alternatives and issues for network signalling transport in a broadband environment', Computer Networks and ISDN Systems, 20, 361-368 (1990).

24. CCITT SGXI, Q.SAAL Draft Recommendation, Geneva, September 1992.

25. CCITT SGXVIII, Draft recommendation I.363, Section 6B-ISDN adaptation layer type 5 (AAL5) specification, Geneva, January 1993.

26. G. 1. Stassinopoulos and I. S. Venieris, 'ATM adaptation 
layer protocols for signalling', Computer Networks and ISDN Systems, 23, 287-304, (1992).

\section{Authors' biographies:}

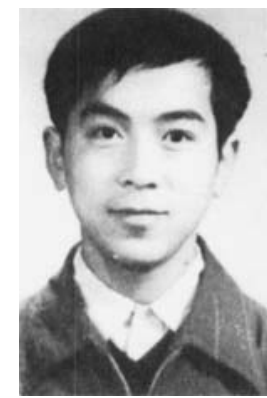

Xinli Hou was born in Xi'an,China on 19 April 1964. He received the B.Sc. and M.Sc. degrees in computer engineering from Xi'an Jiaotong University, Xi'an, China, in 1984 and 1987, respectively. He also received an M.E.E. degree from Philips International Institute, Eindhoven, the Netherlands, in 1990 . He is currently a research staff member of the Centre for Telematics and Information Technology at the University of Twerite, Enschede, the Netherlands, pursuing a Ph.D. degree. His interests are broadband networking, traffic modelling and performance analysis. His email address is xinli@cs.utwente.nl.

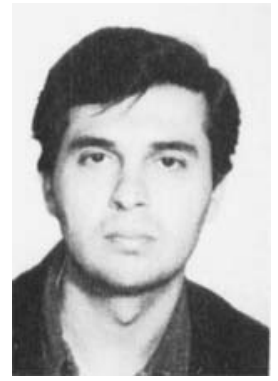

Nikos D. Kalogeropoulos was born in Kyparissia, Greece, on 29 July 1967. He received the Dipl.-Ing. degree from the Electrical and Computer Engineering Department of the National Technical University of Athens (NTUA), Athens, Greece in 1991. He is currently working towards a Ph.D. in the Computer Science Division of NTUA in the area of BISDN access. His work involves multimedia service engineering, object-oriented techniques for signalling systems and network management, access control and performance evaluation for integrated services networks. He is currently involved in sev eral European research projects in telecommunications. $\mathrm{He}$ is a member of the Technical Chamber of Greece.

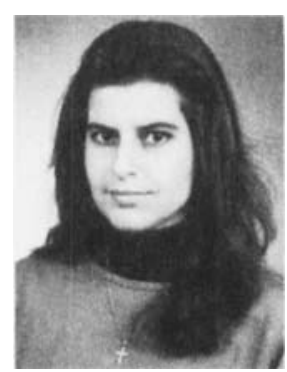

Maria E. Lekkou was born in Athens, Greece, on 21 October 1969. She received the Dipl.-Ing. degree from the Department of Electrical and Computer Engineering, National Technical University of Athens, Athens, Greece, in October 1992. During her studies she received three national scholarships. She is currently working towards a Ph.D. in the Computer Science Division of the National Technical University of Athens, in the area of integrated broadband communications networks (IBCN). Her research interests include signalling protocols for multimedia services, computer-aided modelling and analysis of communication systems, queueing theory and object-ori- ented techniques. She is currently participating in several RACE projects. Mrs. Lekkou is a member of the Technical Chamber of Greece.

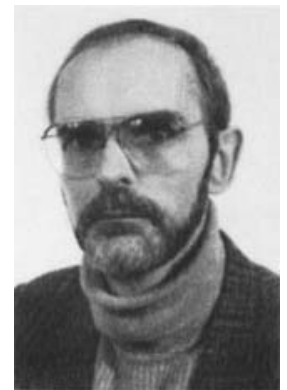

Ignas G. Niemegeers was born in Gent, Belgium in 1947. He received a degree in electrical engineering from the Rijksuniversiteit Gent in 1970. In 1972 he received a M.Sc. E. degree in computer engineering and in 1978 a $\mathrm{Ph} . \mathrm{D}$. degree from Purdue University in West Lafayette, Indiana, USA. From 1978 to 1981 he was a designer of packet switching networks at Bell Telephone Mfg. Cy., Antwerp Belgium. Since 1981 he has been a Professor in the Electrical Engineering and Computer Science Departments of the University of Twente, Enschede, the Netherlands. $\mathrm{He}$ is at present Scientific Director of the Centre for Telematics and Information Technology of the University of Twente. His areas of interest are communication systems and performance analysis. He is active in research on integrated networking, high-speed networking, BISDN, optical switching, performance analysis and performability.

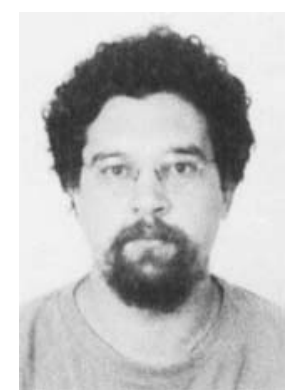

Iakovos $S$. Venieris was born in Naxos, Greece, on 3 March 1965 He received the Dipl.-Ing. degree from the Univesity of Patras, Patras, Greece, in 1988, and the Ph.D. degree from the National Technical University of Athens (NTUA), Athens, Greece, in 1990, all in electrical and computer engineering. During 1991-1992 he was with the National Defense Research Centre, Athens, Greece, performing research in the area of telecommunications electronics for military applications. From January 1988 he joined the Telecommunications Laboratory of NTUA, where he is now a research associate. His research interests are in the fields of BISDN, high speed LANs and MANs, all optical networks, internetworking, signalling, resource scheduling and allocation for network management, modelling, performance evaluation and queueing theory. He has over thirty publications in the above areas. Dr. Venieris has received several national and international awards for academic achievement. He has been exposed to standardization body work and has contributed to ETSI and ITU TSS. He leads NTUA participation in several European Union projects dealing with BISDN protocols, ATM switching and access techniques. He is a reviewer for the IEEE Transactions on Communications, the IEEE Journal on Selected Areas in Communications and the IEEE Communications Magazine. Dr. Venieris is a member of the IEEE and the Technical Chamber of Greece. 